\title{
Machine learning approaches revealed metabolic signatures of incident chronic kidney disease in persons with pre- and type 2 diabetes
}

\section{Supplementary Tables}

\section{Table S1. Metabolite panel of baseline KORA F4 study}

The abbreviations and biochemical names of 163 metabolites are shown in the first and second column, respectively. The third column shows the missing rate of each metabolite among 3,061 KORA F4 individuals. The non-detectable rate was defined as the number of the non-detectable values divided by the number of all measured values. The fourth column presents the arithmetic means of the coefficients of variance $(\mathrm{CV})$ of 114 quality controls samples (i.e. three on each kit plate). The percentage of individuals above the limit of detection (LOD) among 3,061 KORA F4 participants is shown in the fifth column. The sixth column presents the mean value of metabolite concentration $(\mu \mathrm{M})$ in 3,061 KORA F4 participants after adjusting for plate effects. The last column shows the status (used/excluded) for each metabolite.

\begin{tabular}{|c|c|c|c|c|c|c|}
\hline Metabolite & Biochemical name & $\begin{array}{c}\text { Non- } \\
\text { Detectable } \\
\text { Rate }(\%)\end{array}$ & $\begin{array}{l}\mathrm{CV} \\
(\%)\end{array}$ & $\begin{array}{c}\text { Above } \\
\text { LOD }(\%)\end{array}$ & $\begin{array}{c}\text { Mean } \\
\text { Concentration } \\
(\mu \mathrm{M})\end{array}$ & Application \\
\hline $\mathrm{C} 0$ & Carnitine & 0.0 & 7.50 & 99.97 & 35.89 & Used \\
\hline $\mathrm{C} 10$ & Decanoylcarnitine & 0.0 & 12.40 & 98.30 & 0.36 & Used \\
\hline C10:1 & Decenoylcarnitine & 0.0 & 10.45 & 36.20 & 0.17 & Excluded \\
\hline $\mathrm{C} 10: 2$ & Decadienylcarnitine & 0.0 & 15.61 & 58.58 & 0.04 & Used \\
\hline $\mathrm{C} 12$ & Dodecanoylcarnitine & 0.0 & 10.63 & 89.51 & 0.13 & Used \\
\hline $\mathrm{C} 12: 1$ & Dodecenoylcarnitine & 0.0 & 13.51 & 2.16 & 0.15 & Excluded \\
\hline C12-DC & Dodecanedioylcarnitine & 0.0 & 15.71 & 0.00 & 0.06 & Excluded \\
\hline $\mathrm{C} 14$ & Tetradecanoylcarnitine & 0.0 & 11.80 & 47.60 & 0.05 & Excluded \\
\hline C14:1 & Tetradecenoylcarnitine & 0.0 & 20.10 & 99.97 & 0.15 & Used \\
\hline C14:1-OH & Hydroxytetradecenoylcarnitine & 0.0 & 17.88 & 76.54 & 0.02 & Used \\
\hline $\mathrm{C} 14: 2$ & Tetradecadienylcarnitine & 0.0 & 11.19 & 99.44 & 0.03 & Used \\
\hline $\mathrm{C} 14: 2-\mathrm{OH}$ & Hydroxytetradecadienylcarnitine & 0.0 & 24.24 & 44.10 & 0.01 & Excluded \\
\hline $\mathrm{C} 16$ & Hexadecanoylcarnitine & 0.0 & 10.02 & 99.97 & 0.12 & Used \\
\hline C16:1 & Hexadecenoylcarnitine & 0.0 & 10.39 & 2.48 & 0.04 & Excluded \\
\hline $\mathrm{C} 16: 1-\mathrm{OH}$ & Hydroxyhexadecenoylcarnitine & 0.0 & 17.20 & 1.31 & 0.01 & Excluded \\
\hline $\mathrm{C} 16: 2$ & Hexadecadienylcarnitine & 0.0 & 19.46 & 77.56 & 0.01 & Used \\
\hline $\mathrm{C} 16: 2-\mathrm{OH}$ & Hydroxyhexadecadienylcarnitine & 0.0 & 20.19 & 1.08 & 0.01 & Excluded \\
\hline $\mathrm{C} 16-\mathrm{OH}$ & Hydroxyhexadecanoylcarnitine & 0.0 & 21.99 & 3.23 & 0.01 & Excluded \\
\hline $\mathrm{C} 18$ & Octadecanoylcarnitine & 0.0 & 12.52 & 99.90 & 0.05 & Used \\
\hline C18:1 & Octadecenoylcarnitine & 0.0 & 13.30 & 99.93 & 0.13 & Used \\
\hline C18:1-OH & Hydroxyoctadecenoylcarnitine & 0.0 & 25.50 & 1.14 & 0.01 & Excluded \\
\hline $\mathrm{C} 18: 2$ & Octadecadienylcarnitine & 0.0 & 11.00 & 99.97 & 0.05 & Used \\
\hline $\mathrm{C} 2$ & Acetylcarnitine & 0.0 & 9.62 & 99.97 & 8.26 & Used \\
\hline $\mathrm{C} 3$ & Propionylcarnitine & 0.0 & 10.28 & 99.97 & 0.40 & Used \\
\hline $\mathrm{C} 3: 1$ & Propenonylcarnitine & 0.0 & 37.84 & 0.49 & 0.01 & Excluded \\
\hline $\mathrm{C} 3-\mathrm{OH}$ & Hydroxypropionylcarnitine & 0.0 & 98.90 & 7.64 & 0.03 & Excluded \\
\hline $\mathrm{C} 4$ & Butyrylcarnitine & 0.0 & 11.20 & 99.97 & 0.23 & Used \\
\hline $\mathrm{C} 4: 1$ & Butenylcarnitine & 0.0 & 35.99 & 10.42 & 0.02 & Excluded \\
\hline C4-OH (C3-DC) & Hydroxybutyrylcarnitine & 0.0 & 34.81 & 9.64 & 0.09 & Excluded \\
\hline $\mathrm{C} 5$ & Valerylcarnitine & 0.0 & 15.83 & 99.97 & 0.12 & Used \\
\hline $\mathrm{C} 5: 1$ & Tiglylcarnitine & 0.0 & 26.40 & 1.83 & 0.03 & Excluded \\
\hline C5:1-DC & Glutaconylcarnitine & 0.0 & 51.54 & 13.92 & 0.02 & Excluded \\
\hline C5-DC (C6-OH) & Glutarylcarnitine & 0.0 & 36.29 & 58.05 & 0.03 & Excluded \\
\hline C5-M-DC & Methylglutarylcarnitine & 0.0 & 48.62 & 3.82 & 0.03 & Excluded \\
\hline C5-OH (C3-DC- & Hydroxyvalerylcarnitine & 0.0 & 24.31 & 14.05 & 0.04 & Excluded \\
\hline C6 (C4:1-DC) & Hexanoylcarnitine (Fumarylcarnitine) & 0.0 & 14.19 & 87.62 & 0.07 & Used \\
\hline C6:1 & Hexenoylcarnitine & 0.0 & 36.13 & 3.50 & 0.02 & Excluded \\
\hline C7-DC & Pimelylcarnitine & 0.0 & 29.31 & 73.21 & 0.05 & Excluded \\
\hline $\mathrm{C} 8$ & Octanoylcarnitine & 0.0 & 9.73 & 50.38 & 0.23 & Used \\
\hline
\end{tabular}


Glutamine

Glycine

Ornithine

Phenylalanine

14.82

99.97

0.0

Proline

11.33

99.97

99.97

Serine

Threonine

Tryptophan

10.15

100.00

0.0

0.0

9.34

99.97

11.20

99.97

Tyrosine

7.45

99.97

Valine

Leucine/Isoleucine

$\begin{array}{cc}8.61 & 99.97 \\ 15.51 & 100.00\end{array}$

$9.48 \quad 100.00$

Phosphatidylcholine diacyl C24:0

Phosphatidylcholine diacyl C26:0

Phosphatidylcholine diacyl C28:1

Phosphatidylcholine diacyl C30:0

Phosphatidylcholine diacyl C30:2

Phosphatidylcholine diacyl C32:0

Phosphatidylcholine diacyl C32:1

Phosphatidylcholine diacyl C32:2

Phosphatidylcholine diacyl C32:3

Phosphatidylcholine diacyl C34:1

Phosphatidylcholine diacyl C34:2

Phosphatidylcholine diacyl C34:3

Phosphatidylcholine diacyl C34:4

Phosphatidylcholine diacyl C36:0

Phosphatidylcholine diacyl C36:1

Phosphatidylcholine diacyl C36:2

Phosphatidylcholine diacyl C36:3

Phosphatidylcholine diacyl C36:4

Phosphatidylcholine diacyl C36:5

Phosphatidylcholine diacyl C36:6

Phosphatidylcholine diacyl C38:0

Phosphatidylcholine diacyl C38:1

Phosphatidylcholine diacyl C38:3

Phosphatidylcholine diacyl C38:4

Phosphatidylcholine diacyl C38:5

Phosphatidylcholine diacyl C38:6

Phosphatidylcholine diacyl C40:1

Phosphatidylcholine diacyl C40:2

Phosphatidylcholine diacyl C40:3

Phosphatidylcholine diacyl C40:4

Phosphatidylcholine diacyl C40:5

Phosphatidylcholine diacyl C40:6

Phosphatidylcholine diacyl C42:0

Phosphatidylcholine diacyl C42:1

Phosphatidylcholine diacyl C42:2

Phosphatidylcholine diacyl C42:4

Phosphatidylcholine diacyl C42:5

Phosphatidylcholine diacyl C42:6

24.13

78.93

38.23

9.78

11.43

99.97

99.97

99.87

619.01

307.70

98.28

32.03

81.47

62.25

176.09

128.46

106.03

82.62

85.47

277.00

213.92

0.15

1.08

3.38

4.74

75.42

0.06

99.97

$\begin{array}{ll}0.0 & 12.23 \\ 0.0 & 12.32\end{array}$

99.97

20.80

99.97

$\begin{array}{ll}0.0 & 9.92\end{array}$

$0.0 \quad 11.63$

99.97

99.97

16.87

99.97

$0.0 \quad 14.83$

99.97

10.15

99.97

19.81

9.14

99.97

99.97

8.32

99.97

10.63

99.97

11.24

100.00

13.45

99.97

15.22

99.97

99.97

15.09

99.93

19.94

99.97

$\begin{array}{lll}0.0 & 6.64 & 99.97 \\ 0.0 & 9.96 & 99.97\end{array}$

$\begin{array}{lll}0.0 & 6.64 & 99.97 \\ 0.0 & 9.96 & 99.97\end{array}$

$\begin{array}{lll}0.0 & 9.96 & 99.97 \\ 0.0 & 10.27 & 99.97\end{array}$

$0.0 \quad 15.62$

$0.0 \quad 13.75$

9.05

99.97

12.85

99.97

100.00

$0.0 \quad 7.60$

$0.0 \quad 6.43$

$0.0 \quad 6.22$

99.97

100.00

13.59

99.97

$0.0 \quad 15.38$

99.97

15.10

99.97

12.77

99.97

99.97

10.85

62.53

31.78

Phosphatidylcholine acyl-alkyl C30:0

Phosphatidylcholine acyl-alkyl C30:1

Phosphatidylcholine acyl-alkyl C30:2

Phosphatidylcholine acyl-alkyl C32:1

Phosphatidylcholine acyl-alkyl C32:2

Phosphatidylcholine acyl-alkyl C34:0

Phosphatidylcholine acyl-alkyl C34:1

Phosphatidylcholine acyl-alkyl C34:2

46.30

99.71

98.66

92.22

99.97

10.34

99.97

12.20

11.28

99.97

99.97

15.21

21.98

3.95

0.48

240.68

392.77

18.07

2.27

2.72

53.89

232.62

150.39

220.61

29.52

1.13

3.29

0.87

54.08

119.83

62.43

90.66

0.47

0.36

0.66

4.17

11.53

28.76

0.60

0.30

0.21

0.22

0.43

0.63

0.48

0.24

0.16

2.85

0.75

1.73

$0.0 \quad 11.88$

10.56

12.38

99.97

12.67

Used

Excluded

Used

Used

Used

Used

Used

Used

Used

Used

Used

Used

Used

Used

Used

Used

Used

Excluded

Used

Used

Excluded

Used

Used

Used

Used

Used

Used

Used

Used

Used

Used

Used

Used

Used

Used

Used

Used

Used

Used

Used

Used

Used

Excluded

Used

Used

Used

Used

Used

Used

Used

Used

Used

Used

Used 


\begin{tabular}{|c|c|c|c|c|c|c|}
\hline $\mathrm{PC}$ ae $\mathrm{C} 34: 3$ & Phosphatidylcholine acyl-alkyl C34:3 & 0.0 & 9.93 & 99.97 & 8.38 & Used \\
\hline $\mathrm{PC}$ ae $\mathrm{C} 36: 0$ & Phosphatidylcholine acyl-alkyl C36:0 & 0.0 & 40.89 & 99.97 & 1.10 & Excluded \\
\hline $\mathrm{PC}$ ae $\mathrm{C} 36: 1$ & Phosphatidylcholine acyl-alkyl C36:1 & 0.0 & 12.61 & 99.97 & 8.40 & Used \\
\hline $\mathrm{PC}$ ae $\mathrm{C} 36: 2$ & Phosphatidylcholine acyl-alkyl C36:2 & 0.0 & 13.72 & 99.97 & 15.19 & Used \\
\hline $\mathrm{PC}$ ae $\mathrm{C} 36: 3$ & Phosphatidylcholine acyl-alkyl C36:3 & 0.0 & 12.59 & 99.97 & 8.59 & Used \\
\hline PC ae C36:4 & Phosphatidylcholine acyl-alkyl C36:4 & 0.0 & 11.60 & 99.97 & 20.88 & Used \\
\hline PC ae C36:5 & Phosphatidylcholine acyl-alkyl C36:5 & 0.0 & 9.39 & 99.97 & 13.85 & Used \\
\hline PC ae C38:0 & Phosphatidylcholine acyl-alkyl C38:0 & 0.0 & 12.57 & 99.97 & 2.48 & Used \\
\hline PC ae C38:1 & Phosphatidylcholine acyl-alkyl C38:1 & 0.0 & 14.05 & 99.97 & 0.82 & Used \\
\hline $\mathrm{PC}$ ae $\mathrm{C} 38: 2$ & Phosphatidylcholine acyl-alkyl C38:2 & 0.0 & 13.49 & 99.97 & 2.15 & Used \\
\hline $\mathrm{PC}$ ae $\mathrm{C} 38: 3$ & Phosphatidylcholine acyl-alkyl C38:3 & 0.0 & 10.85 & 99.97 & 4.34 & Used \\
\hline $\mathrm{PC}$ ae $\mathrm{C} 38: 4$ & Phosphatidylcholine acyl-alkyl C38:4 & 0.0 & 12.38 & 99.97 & 15.73 & Used \\
\hline $\mathrm{PC}$ ae $\mathrm{C} 38: 5$ & Phosphatidylcholine acyl-alkyl C38:5 & 0.0 & 11.10 & 100.00 & 19.96 & Used \\
\hline PC ae C38:6 & Phosphatidylcholine acyl-alkyl C38:6 & 0.0 & 9.18 & 99.97 & 8.70 & Used \\
\hline PC ae C40:0 & Phosphatidylcholine acyl-alkyl C40:0 & 0.0 & 8.03 & 1.14 & 10.25 & Excluded \\
\hline $\mathrm{PC}$ ae $\mathrm{C} 40: 1$ & Phosphatidylcholine acyl-alkyl C40:1 & 0.0 & 12.62 & 99.97 & 1.68 & Used \\
\hline $\mathrm{PC}$ ae $\mathrm{C} 40: 2$ & Phosphatidylcholine acyl-alkyl C40:2 & 0.0 & 11.32 & 99.97 & 2.10 & Used \\
\hline $\mathrm{PC}$ ae $\mathrm{C} 40: 3$ & Phosphatidylcholine acyl-alkyl C40:3 & 0.0 & 10.64 & 99.97 & 1.14 & Used \\
\hline $\mathrm{PC}$ ae $\mathrm{C} 40: 4$ & Phosphatidylcholine acyl-alkyl C40:4 & 0.0 & 10.30 & 99.97 & 2.59 & Used \\
\hline $\mathrm{PC}$ ae $\mathrm{C} 40: 5$ & Phosphatidylcholine acyl-alkyl C40:5 & 0.0 & 8.88 & 99.97 & 3.57 & Used \\
\hline PC ae C40:6 & Phosphatidylcholine acyl-alkyl C40:6 & 0.0 & 11.23 & 99.97 & 5.06 & Used \\
\hline $\mathrm{PC}$ ae $\mathrm{C} 42: 0$ & Phosphatidylcholine acyl-alkyl C42:0 & 0.0 & 18.33 & 14.80 & 0.52 & Excluded \\
\hline $\mathrm{PC}$ ae $\mathrm{C} 42: 1$ & Phosphatidylcholine acyl-alkyl C42:1 & 0.0 & 13.91 & 99.97 & 0.38 & Used \\
\hline $\mathrm{PC}$ ae $\mathrm{C} 42: 2$ & Phosphatidylcholine acyl-alkyl C42:2 & 0.0 & 17.58 & 99.97 & 0.68 & Used \\
\hline $\mathrm{PC}$ ae $\mathrm{C} 42: 3$ & Phosphatidylcholine acyl-alkyl C42:3 & 0.0 & 11.87 & 99.97 & 0.87 & Used \\
\hline $\mathrm{PC}$ ae $\mathrm{C} 42: 4$ & Phosphatidylcholine acyl-alkyl C42:4 & 0.0 & 9.99 & 100.00 & 1.01 & Used \\
\hline $\mathrm{PC}$ ae $\mathrm{C} 42: 5$ & Phosphatidylcholine acyl-alkyl C42:5 & 0.0 & 7.27 & 99.93 & 2.36 & Used \\
\hline $\mathrm{PC}$ ae $\mathrm{C} 44: 3$ & Phosphatidylcholine acyl-alkyl C44:3 & 0.0 & 13.32 & 99.97 & 0.11 & Used \\
\hline $\mathrm{PC}$ ae $\mathrm{C} 44: 4$ & Phosphatidylcholine acyl-alkyl C44:4 & 0.0 & 11.71 & 99.97 & 0.43 & Used \\
\hline $\mathrm{PC}$ ae $\mathrm{C} 44: 5$ & Phosphatidylcholine acyl-alkyl C44:5 & 0.0 & 7.15 & 99.97 & 2.12 & Used \\
\hline PC ae C44:6 & Phosphatidylcholine acyl-alkyl C44:6 & 0.0 & 7.73 & 99.97 & 1.38 & Used \\
\hline lysoPC a C14:0 & lysoPhosphatidylcholine acyl C14:0 & 0.0 & 26.82 & 42.21 & 3.21 & Excluded \\
\hline lysoPC a C16:0 & lysoPhosphatidylcholine acyl C16:0 & 0.0 & 10.69 & 99.97 & 94.07 & Used \\
\hline lysoPC a C16:1 & lysoPhosphatidylcholine acyl C16:1 & 0.0 & 10.01 & 99.97 & 2.90 & Used \\
\hline lysoPC a C17:0 & lysoPhosphatidylcholine acyl C17:0 & 0.0 & 13.05 & 99.97 & 1.72 & Used \\
\hline lysoPC a C18:0 & lysoPhosphatidylcholine acyl C18:0 & 0.0 & 10.27 & 99.97 & 25.96 & Used \\
\hline lysoPC a C18:1 & lysoPhosphatidylcholine acyl C18:1 & 0.0 & 11.29 & 99.97 & 19.22 & Used \\
\hline lysoPC a C18:2 & lysoPhosphatidylcholine acyl C18:2 & 0.0 & 9.42 & 99.97 & 27.22 & Used \\
\hline lysoPC a C20:3 & lysoPhosphatidylcholine acyl C20:3 & 0.0 & 10.95 & 99.97 & 2.38 & Used \\
\hline lysoPC a C20:4 & lysoPhosphatidylcholine acyl C20:4 & 0.0 & 9.34 & 99.97 & 6.77 & Used \\
\hline lysoPC a C24:0 & lysoPhosphatidylcholine acyl C24:0 & 0.0 & 21.21 & 8.04 & 0.36 & Excluded \\
\hline lysoPC a C26:0 & lysoPhosphatidylcholine acyl C26:0 & 0.0 & 32.22 & 59.85 & 0.54 & Excluded \\
\hline lysoPC a C26:1 & lysoPhosphatidylcholine acyl C26:1 & 0.0 & 10.71 & 0.00 & 2.02 & Excluded \\
\hline lysoPC a C28:0 & lysoPhosphatidylcholine acyl C28:0 & 0.0 & 27.17 & 46.46 & 0.48 & Excluded \\
\hline lysoPC a C28:1 & lysoPhosphatidylcholine acyl C28:1 & 0.0 & 22.50 & 99.84 & 0.62 & Used \\
\hline 1ysoPC a C6:0 & lysoPhosphatidylcholine acyl C6:0 & 0.0 & 43.89 & 25.51 & 0.02 & Excluded \\
\hline $\mathrm{SM}(\mathrm{OH}) \mathrm{C} 14: 1$ & Hydroxysphingomyeline C14:1 & 0.0 & 12.85 & 100.00 & 6.18 & Used \\
\hline $\mathrm{SM}(\mathrm{OH}) \mathrm{C} 16: 1$ & Hydroxysphingomyeline C16:1 & 0.0 & 8.72 & 99.97 & 3.35 & Used \\
\hline $\mathrm{SM}(\mathrm{OH}) \mathrm{C} 22: 1$ & Hydroxysphingomyeline C22:1 & 0.0 & 14.23 & 99.97 & 13.43 & Used \\
\hline $\mathrm{SM}(\mathrm{OH}) \mathrm{C} 22: 2$ & Hydroxysphingomyeline C22:2 & 0.0 & 13.12 & 99.97 & 11.40 & Used \\
\hline $\mathrm{SM}(\mathrm{OH}) \mathrm{C} 24: 1$ & Hydroxysphingomyeline C24:1 & 0.0 & 17.05 & 99.97 & 1.34 & Used \\
\hline SM C16:0 & Sphingomyelin C16:0 & 0.0 & 12.92 & 99.97 & 105.98 & Used \\
\hline SM C16:1 & Sphingomyelin C16:1 & 0.0 & 11.64 & 99.97 & 15.97 & Used \\
\hline SM C18:0 & Sphingomyelin C18:0 & 0.0 & 9.29 & 99.97 & 23.16 & Used \\
\hline SM C18:1 & Sphingomyelin C18:1 & 0.0 & 10.86 & 100.00 & 11.25 & Used \\
\hline SM C20:2 & Sphingomyelin C20:2 & 0.1 & 15.99 & 99.97 & 0.38 & Used \\
\hline SM C22:3 & Sphingomyelin C22:3 & 43.6 & 60.99 & 99.51 & 0.22 & Excluded \\
\hline SM C24:0 & Sphingomyelin C24:0 & 0.0 & 14.33 & 99.97 & 21.68 & Used \\
\hline SM C24:1 & Sphingomyelin C24:1 & 0.0 & 15.01 & 100.00 & 52.40 & Used \\
\hline SM C26:0 & Sphingomyelin C26:0 & 0.0 & 57.33 & 99.97 & 0.32 & Excluded \\
\hline SM C26:1 & Sphingomyelin C26:1 & 0.0 & 22.75 & 99.97 & 0.42 & Used \\
\hline H1 & Sum of Hexoses & 0.0 & 6.33 & 99.97 & 5197.44 & Used \\
\hline
\end{tabular}


Table S2. List of 26 metabolites significantly associated with incident chronic kidney disease in either basic or full model in hyperglycemic individuals

Odds ratios $(O R \mathrm{~s})$ with $95 \% C I$ and $P$-values of multivariable logistic regression are shown. The basic model was adjusted for age, sex, BMI, systolic blood pressure, smoking status, triglyceride, total cholesterol, HDL cholesterol, and fasting serum glucose. The full model was additionally adjusted for use of lipid lowering drugs, antihypertensive and anti-diabetic medication, baseline estimated glomerular filtration rate and urinary albumin-to-creatinine ratio. $P$-values shown in bold represent statistical significance at 0.05 level. Abbreviations: SM, sphingomyelin; PC aa, phosphatidylcholine diacyl; PC ae, phosphatidylcholine acyl-alkyl.

\begin{tabular}{|c|c|c|c|c|}
\hline \multirow[t]{2}{*}{ Metabolites } & \multicolumn{2}{|c|}{ Basic Model } & \multicolumn{2}{|c|}{ Full Model } \\
\hline & OR $(95 \% C I)$ & $P$-value & $O R(95 \% C I)$ & $P$-value \\
\hline $\mathrm{C} 10$ & $1.42(1.03-1.98)$ & 3.317E-02 & $1.24(0.86-1.80)$ & $2.495 \mathrm{E}-01$ \\
\hline $\mathrm{C} 12$ & $1.49(1.09-2.05)$ & $1.268 \mathrm{E}-02$ & $1.35(0.95-1.92)$ & $9.131 \mathrm{E}-02$ \\
\hline C14:1 & $1.37(1.04-1.83)$ & 2.919E-02 & $1.36(0.99-1.89)$ & $5.751 \mathrm{E}-02$ \\
\hline $\mathrm{C} 18$ & $1.44(1.06-1.98)$ & 2.331E-02 & $1.30(0.92-1.84)$ & $1.376 \mathrm{E}-01$ \\
\hline C18:1 & $1.44(1.07-1.97)$ & 1.892E-02 & $1.39(0.99-1.96)$ & $6.293 \mathrm{E}-02$ \\
\hline C6 (C4:1-DC) & $1.41(1.05-1.89)$ & 2.244E-02 & $1.25(0.90-1.75)$ & $1.884 \mathrm{E}-01$ \\
\hline $\mathrm{C} 8$ & $1.39(1.02-1.90)$ & 3.948E-02 & $1.21(0.85-1.71)$ & 2.919E-01 \\
\hline Arginine & $1.40(1.07-1.89)$ & 2.154E-02 & $1.25(0.93-1.73)$ & $1.577 \mathrm{E}-01$ \\
\hline Proline & $1.38(1.01-1.89)$ & 4.453E-02 & $1.39(0.98-1.97)$ & $6.337 \mathrm{E}-02$ \\
\hline PC aa C32:2 & $0.72(0.56-0.93)$ & 1.275E-02 & $0.74(0.56-0.99)$ & $3.690 \mathrm{E}-02$ \\
\hline PC aa C38:0 & $1.51(1.12-2.07)$ & 8.059E-03 & $1.56(1.12-2.21)$ & $1.043 \mathrm{E}-02$ \\
\hline PC aa C42:0 & $1.41(1.04-1.92)$ & $2.686 \mathrm{E}-02$ & $1.40(1.01-1.96)$ & 4.801E-02 \\
\hline PC ae C38:6 & $1.41(1.01-1.99)$ & 4.573E-02 & $1.40(0.96-2.06)$ & $8.386 \mathrm{E}-02$ \\
\hline PC ae C40:5 & $1.42(1.04-1.95)$ & 3.009E-02 & $1.32(0.94-1.88)$ & $1.181 \mathrm{E}-01$ \\
\hline PC ae C40:6 & $1.54(1.12-2.14)$ & $9.600 \mathrm{E}-03$ & $1.57(1.10-2.27)$ & $1.358 E-02$ \\
\hline $\mathrm{PC}$ ae $\mathrm{C} 42: 5$ & $1.43(1.06-1.96)$ & 2.234E-02 & $1.29(0.92-1.81)$ & $1.457 \mathrm{E}-01$ \\
\hline SM (OH) C14:1 & $1.50(1.06-2.13)$ & 2.277E-02 & $1.56(1.07-2.32)$ & $2.382 \mathrm{E}-02$ \\
\hline SM (OH) C16:1 & $1.59(1.14-2.24)$ & $6.923 \mathrm{E}-03$ & $1.63(1.14-2.39)$ & $9.614 E-03$ \\
\hline $\mathrm{SM}(\mathrm{OH}) \mathrm{C} 22: 2$ & $1.58(1.09-2.33)$ & 1.880E-02 & $1.50(1.00-2.30)$ & $5.674 \mathrm{E}-02$ \\
\hline SM C16:0 & $1.91(1.29-2.91)$ & 1.811E-03 & $1.82(1.17-2.91)$ & 9.378E-03 \\
\hline SM C16:1 & $1.91(1.29-2.88)$ & 1.557E-03 & $1.85(1.19-2.94)$ & 7.145E-03 \\
\hline SM C18:0 & $1.86(1.34-2.63)$ & 2.839E-04 & $1.80(1.26-2.63)$ & $1.754 \mathrm{E}-03$ \\
\hline SM C18:1 & $2.25(1.54-3.39)$ & 4.976E-05 & $2.22(1.46-3.49)$ & 3.315E-04 \\
\hline SM C20:2 & $1.40(1.05-1.93)$ & 3.045E-02 & $1.51(1.10-2.14)$ & $1.411 \mathrm{E}-02$ \\
\hline SM C24:1 & $1.62(1.15-2.31)$ & $7.066 \mathrm{E}-03$ & $1.57(1.08-2.33)$ & 2.061E-02 \\
\hline SM C26:1 & $1.41(1.05-1.93)$ & 2.564E-02 & $1.57(1.13-2.23)$ & $8.215 E-03$ \\
\hline
\end{tabular}


Table S3. Baseline characteristics of propensity scores matched case-control hyperglycemic individuals

Clinical variables of incident CKD patients (= cases) matched with non-CKD participants (= controls) are shown. Mean \pm standard deviation is provided when appropriate; $P$-values were calculated by univariate conditional logistic regression. $P$-values shown in bold represent statistical significance at 0.05 level. Abbreviations: CKD, chronic kidney disease; eGFR, estimated glomerular filtration rate; UACR, urinary albumin-to-creatinine ratio.

\begin{tabular}{lccc}
\hline Clinical variables & $\begin{array}{c}\text { Incident CKD } \\
\mathbf{N}=\mathbf{6 2}\end{array}$ & $\begin{array}{c}\text { Non-CKD } \\
\mathbf{N}=\mathbf{6 2}\end{array}$ & $\boldsymbol{P}$-value \\
\hline Age, years & $65.81 \pm 9.3$ & $65.48 \pm 7.62$ & 0.777 \\
Sex, Male, $\mathrm{n}(\%)$ & 54.84 & 64.52 & 0.261 \\
BMI, kg/m & $30.53 \pm 4.84$ & $29.79 \pm 3.97$ & 0.335 \\
Fasting glucose, mg/dl & $112.68 \pm 27.31$ & $114.32 \pm 19.32$ & 0.676 \\
Systolic blood pressure, mmHg & $130.03 \pm 19.79$ & $130.83 \pm 16.38$ & 0.819 \\
Triglyceride, mg/dl a & $136.5[99.5-186]$ & $129[93.5-182.75]$ & 0.784 \\
Total cholesterol, mg/dl & $215 \pm 38.05$ & $211 \pm 33.11$ & 0.481 \\
HDL cholesterol, mg/dl & $51.81 \pm 11.59$ & $51.66 \pm 14.29$ & 0.951 \\
eGFR, mL/min/1.73 m ${ }^{2}$ & $80.17 \pm 14.79$ & $81.95 \pm 10.92$ & 0.339 \\
UACR, mg/g a & $8.89[4.44-13.41]$ & $6.8[4.85-14.36]$ & 0.842 \\
Smoking, \% & & & \\
$\quad$ Non-smoker & 43.55 & 41.94 & Reference \\
Former smoker & 50 & 53.23 & 0.704 \\
$\quad$ Current smoke & 6.45 & 4.84 & 0.729 \\
Medication usage, \% & & & \\
$\quad$ Lipid-lowering & 19.35 & 25.81 & 0.396 \\
$\quad$ Antihypertensive & 62.9 & 61.29 & 0.842 \\
$\quad$ Anti-diabetic & 14.52 & 16.13 & 0.796 \\
\hline
\end{tabular}

${ }^{\mathrm{a}}$ values are presented as median [25th- 75 th percentile].

Table S4. Results of sensitivity analyses - the two metabolites significantly associated with incident chronic kidney disease in the propensity scores matched case-control hyperglycemic individuals

Odds ratios $(O R \mathrm{~s})$ per standard deviation (SD) with 95\% CI and $P$-values of conditional logistic regression results are shown. $P$-values shown in bold represent statistical significance at 0.05 level. Abbreviations: SM, sphingomyelin; PC aa, phosphatidylcholine diacyl.

\begin{tabular}{lcc}
\hline & SM C18:1 & PC aa C38:0 \\
\hline OR $(95 \% C I)$, per SD & $1.77(1.14-2.73)$ & $1.71(1.12-2.62)$ \\
$P$ - value & 0.011 & 0.014 \\
\hline
\end{tabular}


Table S5. Results of sensitivity analyses - interaction effects of the two metabolites with different glucose subgroups

Odds ratios $(O R \mathrm{~s})$ with $95 \% C I$ and $P$-values of multivariate logistic regression results are shown. $P_{\text {interaction }}$ represents $P$-value of multiplicative interaction effects between metabolite and different glucose groups. $P$-values shown in bold represent statistical significance at 0.05 level. Abbreviations: SM, sphingomyelin; PC aa, phosphatidylcholine diacyl; NGT, normal glucose tolerance; 2-h glucose, two hour post load glucose.

\begin{tabular}{|c|c|c|c|c|c|c|}
\hline \multirow[b]{2}{*}{ Group } & \multicolumn{4}{|c|}{ SM C18:1 } & \multicolumn{2}{|c|}{ PC aa C38:0 } \\
\hline & $\begin{array}{c}\text { OR } \\
(95 \% C I)\end{array}$ & $P$ - values & $\boldsymbol{P}_{\text {interaction }}$ & $\begin{array}{c}O R \\
(95 \% C I)\end{array}$ & $P$ - values & $\boldsymbol{P}_{\text {interaction }}$ \\
\hline Glycemic status & & & $1.774 \mathrm{E}-03^{\mathrm{c}}$ & & & $0.417^{c}$ \\
\hline $\mathrm{NGT}^{\mathrm{a}}$ & $\begin{array}{c}0.76 \\
(0.57-1.01)\end{array}$ & 0.057 & & $\begin{array}{c}1.21 \\
(0.95-1.55)\end{array}$ & 0.124 & \\
\hline Hyperglycemia $^{\mathrm{b}}$ & $\begin{array}{c}2.22 \\
(1.46-3.49) \\
\end{array}$ & 3.315E-04 & & $\begin{array}{c}1.56 \\
(1.12-2.21) \\
\end{array}$ & 0.010 & \\
\hline Fasting glucose & & & $0.241^{\mathrm{d}}$ & & & $0.609^{\mathrm{d}}$ \\
\hline 1st tertile ${ }^{\mathrm{a}}$ & $\begin{array}{c}0.78 \\
(0.46-1.36)\end{array}$ & 0.372 & & $\begin{array}{c}1.13 \\
(0.73-1.77)\end{array}$ & 0.579 & \\
\hline 2nd tertile ${ }^{a}$ & $\begin{array}{c}0.84 \\
(0.56-1.27)\end{array}$ & 0.412 & & $\begin{array}{c}1.33 \\
(0.94-1.88)\end{array}$ & 0.106 & \\
\hline Top tertile & $\begin{array}{c}1.50 \\
(1.08-2.11) \\
\end{array}$ & 0.019 & & $\begin{array}{c}1.49 \\
(1.10-2.03) \\
\end{array}$ & 0.010 & \\
\hline 2-h glucose & & & $0.010^{\mathrm{e}}$ & & & $0.538^{\mathrm{e}}$ \\
\hline 1 st tertile & $\begin{array}{c}0.55 \\
(0.33-0.92)\end{array}$ & 0.023 & & $\begin{array}{c}1.22 \\
(0.79-1.87)\end{array}$ & 0.369 & \\
\hline 2nd tertile ${ }^{a}$ & $\begin{array}{c}0.74 \\
(0.48-1.14)\end{array}$ & 0.172 & & $\begin{array}{c}1.27 \\
(0.87-1.88)\end{array}$ & 0.231 & \\
\hline Top tertile & $\begin{array}{c}1.58 \\
(1.07-2.37) \\
\end{array}$ & 0.022 & & $\begin{array}{c}1.60 \\
(1.17-2.23) \\
\end{array}$ & 0.004 & \\
\hline
\end{tabular}

${ }^{a}$ with adjustments for age, sex, BMI, systolic blood pressure, smoking status, triglyceride, total cholesterol, HDL cholesterol, fasting glucose, use of lipid lowering drugs, antihypertensive medication, baseline estimated glomerular filtration rate and baseline urinary albumin-to-creatinine ratio.

${ }^{\mathrm{b}}$ with adjustment for the covariates shown in ${ }^{\mathrm{a}}$ as well as use of anti-diabetic medication.

${ }^{\mathrm{c}}$ The model setting : $\operatorname{logit}(P)=\beta_{0}+\beta_{1} *$ metabolite $+\beta_{2} *$ glycemic status $+\beta_{3} *$ metabolite $*$ glycemic status $+\beta_{4} *$ covariates + $\varepsilon$. The covariates including the covariates shown in a well as use of anti-diabetic medication.

$\mathrm{d}$ The model setting : $\operatorname{logit}(P)=\beta_{0}+\beta_{1} *$ metabolite $+\beta_{2} *$ three tertiles group of fasting glucose $+\beta_{3} *$ metabolite $*$ three tertiles group of fasting glucose $+\beta_{4} *$ covariates $+\varepsilon$. The covariates included the covariates shown in as well as use of anti-diabetic medication except fasting glucose.

e The model setting : $\operatorname{logit}(P)=\beta_{0}+\beta_{1} *$ metabolite $+\beta_{2} *$ three tertiles group of 2 -h glucose $+\beta_{3} *$ metabolite $*$ three tertiles group of 2-h glucose $+\beta_{4}$ *covariates $+\varepsilon$. The covariates included the covariates shown in a except fasting glucose. 
Table S6. Results of sensitivity analyses - association of two candidate biomarkers with UACRand eGFR- based incident CKD in hyperglycemic participants

Odds ratios $(O R \mathrm{~s})$ with $95 \% \mathrm{CI}$ and $P$-values of each metabolite with UACR-based and eGFR-based incident CKD in basic and full multivariable logistic regression models are shown, respectively. UACRbased incident CKD was defined as UACR $\geq 30 \mathrm{mg} / \mathrm{g}$ at follow-up (FF4). eGFR-based incident CKD was defined as eGFR $<60 \mathrm{ml} / \mathrm{min} / 1.73 \mathrm{~m}^{2}$ at follow-up (FF4). Basic model was adjusted for age, sex, BMI, systolic blood pressure, smoking status, triglyceride, total cholesterol, HDL cholesterol and fasting glucose. Full model was additionally adjusted for use of lipid lowering drugs, antihypertensive and antidiabetic medication, baseline eGFR and UACR. $P$-values shown in bold represent statistical significance at 0.05 level. Abbreviations: CKD, chronic kidney disease; eGFR, estimated glomerular filtration rate; UACR, urinary albumin-to-creatinine ratio; SM, sphingomyelin; PC aa, phosphatidylcholine diacyl.

\begin{tabular}{|c|c|c|c|c|}
\hline & \multicolumn{2}{|c|}{ SM C18:1 } & \multicolumn{2}{|c|}{ PC aa C38:0 } \\
\hline & Basic model & Full model & Basic model & Full model \\
\hline \multicolumn{5}{|c|}{ UACR- based incident CKD $(\mathrm{N}=32) \&$ non-CKD $(\mathrm{N}=353)$} \\
\hline$P$-value & 0.024 & 0.040 & 0.022 & 0.004 \\
\hline OR $(95 \% C I)$, per SD & $1.79(1.10-3.03)$ & $1.80(1.05-3.25)$ & $1.66(1.08-2.58)$ & $2.17(1.31-3.76)$ \\
\hline \multicolumn{5}{|c|}{ eGFR- based incident CKD $(\mathrm{N}=65) \&$ non-CKD $(\mathrm{N}=320)$} \\
\hline$P$-value & 0.008 & 0.107 & 0.061 & 0.247 \\
\hline OR $(95 \% C I)$, per SD & $1.77(1.17-2.75)$ & $1.50(0.93-2.5)$ & $1.38(0.99-1.94)$ & $1.25(0.86-1.85)$ \\
\hline
\end{tabular}

Table S7. Comparison of the predictive performances of two sets of predictors of incident chronic kidney disease in hyperglycemic individuals with three machine learning approaches

The median AUC (95\% CI) of three machine learning approaches over 100 random repeats of 10-fold cross validation are shown. Reference predictors consists of baseline age, sex, estimated glomerular filtration rate and urinary albumin-to-creatinine ratio. Developed sets includes combined metabolites and clinical variables that were selected by the three-step feature selection in each round. Abbreviation: AUC, area under the receiver operating characteristic curve.

\begin{tabular}{cllcc}
\hline Algorithms & \multicolumn{1}{c}{ Models } & \multicolumn{1}{c}{$\begin{array}{c}\text { Median } \\
\text { AUC (95\% CI) }\end{array}$} & $\begin{array}{c}\text { Absolute } \\
\text { increase in } \\
\text { median } \\
\text { prediction }\end{array}$ & $\begin{array}{c}\text { Outperform } \\
\text { times over } \\
\mathbf{1 0 0} \text { times }\end{array}$ \\
\hline $\begin{array}{c}\text { Support } \\
\text { Vector Machine }\end{array}$ & $\begin{array}{l}\text { Reference predictors } \\
\text { Developed sets }\end{array}$ & $0.800(0.783-0.816)$ & $2.5 \%$ & 97 \\
\hline $\begin{array}{c}\text { Random } \\
\text { Forest }\end{array}$ & Reference predictors & $0.825(0.801-0.849)$ & \multirow{2}{*}{100} \\
\hline $\begin{array}{c}\text { Adaptive } \\
\text { Boosting }\end{array}$ & Reveloped sets & $0.818(0.794-0.836)$ & $2.9 \%$ & 87 \\
\hline
\end{tabular}


Table S8. The total selected times for three most frequently selected sets of metabolites and clinical variables over 1000 selection rounds in 100 times of $\mathbf{1 0}$-fold cross validation

The three most frequently selected sets of metabolites and clinical variables, as well as their total selected times over 1000 selection rounds are shown. Abbreviations: eGFR, estimated glomerular filtration rate; UACR, urinary albumin-to-creatinine ratio; SM, sphingomyelin; PC aa, phosphatidylcholine diacyl.

\begin{tabular}{cc}
\hline Sets of metabolites and clinical variables & Selected times \\
\hline SM C18:1, PC aa C38:0, age, total cholesterol, fasting glucose, eGFR, UACR & 113 \\
SM C18:1, age, total cholesterol, fasting glucose, eGFR, UACR & 78 \\
SM C18:1, PC aa C38:0, proline, age, total cholesterol, fasting glucose, eGFR, UACR & 67 \\
\hline
\end{tabular}

Table S9. The selected times for 15 most important variables over 1000 selection rounds in 100 times of 10-fold cross validation

Out of 125 metabolites and 14 clinical variables, 15 most frequently selected variables and their total selected times over 1000 selection rounds are shown. Abbreviations: UACR, urinary albumin-tocreatinine ratio; eGFR, estimated glomerular filtration rate; SM, sphingomyelin; PC aa, phosphatidylcholine diacyl.

\begin{tabular}{cc}
\hline Variables & Selected times \\
\hline UACR & 1000 \\
eGFR & 1000 \\
Age & 999 \\
Total cholesterol & 996 \\
Fasting glucose & 942 \\
SM C18:1 & 857 \\
PC aa C38:0 & 593 \\
Triglyceride & 270 \\
Proline & 229 \\
PC aa C32:2 & 156 \\
Tyrosine & 129 \\
SM C26:1 & 109 \\
C18:1 & 108 \\
PC aa C36:4 & 92 \\
Use of lipid lowering drugs & 81
\end{tabular}


Table S10. Predictive performance of the best set of predictors and the full model of incident CKD in hyperglycemia

Mean AUC values of the best set of predictors and the full model of incident CKD in hyperglycemia are shown. The mean AUC value of the best set of predictors was the average value of the AUC values of the 113 selected times, in which the models were fitted with support vector machine. The average AUC value of the full model was obtained using logistic regression with 10 times of 10-fold cross validation. Abbreviations: CKD, chronic kidney disease; AUC, area under the receiver operating characteristic curve; UACR, urinary albumin-to-creatinine ratio; eGFR, estimated glomerular filtration rate; SM, sphingomyelin; PC aa, phosphatidylcholine diacyl.

\begin{tabular}{|c|c|c|}
\hline Models & Mean AUC & $\begin{array}{l}\text { Absolute increase in } \\
\text { mean prediction }\end{array}$ \\
\hline $\begin{array}{c}\text { The best set of predictors } \\
\text { (i.e., SM C18:1, PC aa C38: 0, age, total cholesterol, fasting glucose, } \\
\text { eGFR and UACR) }\end{array}$ & 0.857 & \\
\hline $\begin{array}{l}\text { The full model } \\
\text { (i.e., age, sex, BMI, systolic blood pressure, smoking status, } \\
\text { triglyceride, total cholesterol, HDL cholesterol, fasting glucose, use of } \\
\text { lipid lowering drugs, antihypertensive and anti-diabetic medication, } \\
\text { eGFR and UACR }\end{array}$ & 0.809 & $4.8 \%$ \\
\hline
\end{tabular}




\section{Supplementary Figures}

\section{Figure S1. Technical normalization across the study}

Comparison of before and after normalization of plate effect of metabolite data using phosphatidylcholine diacyl (PC aa) $\mathrm{C} 34: 2$ as an example. Metabolite concentration drifts at 38 plates were independently corrected by conducting plate effect normalization in quality controls samples (QCs, shown in plots A and B) and KORA F4 individual samples (plots C and D).

Fig. S1
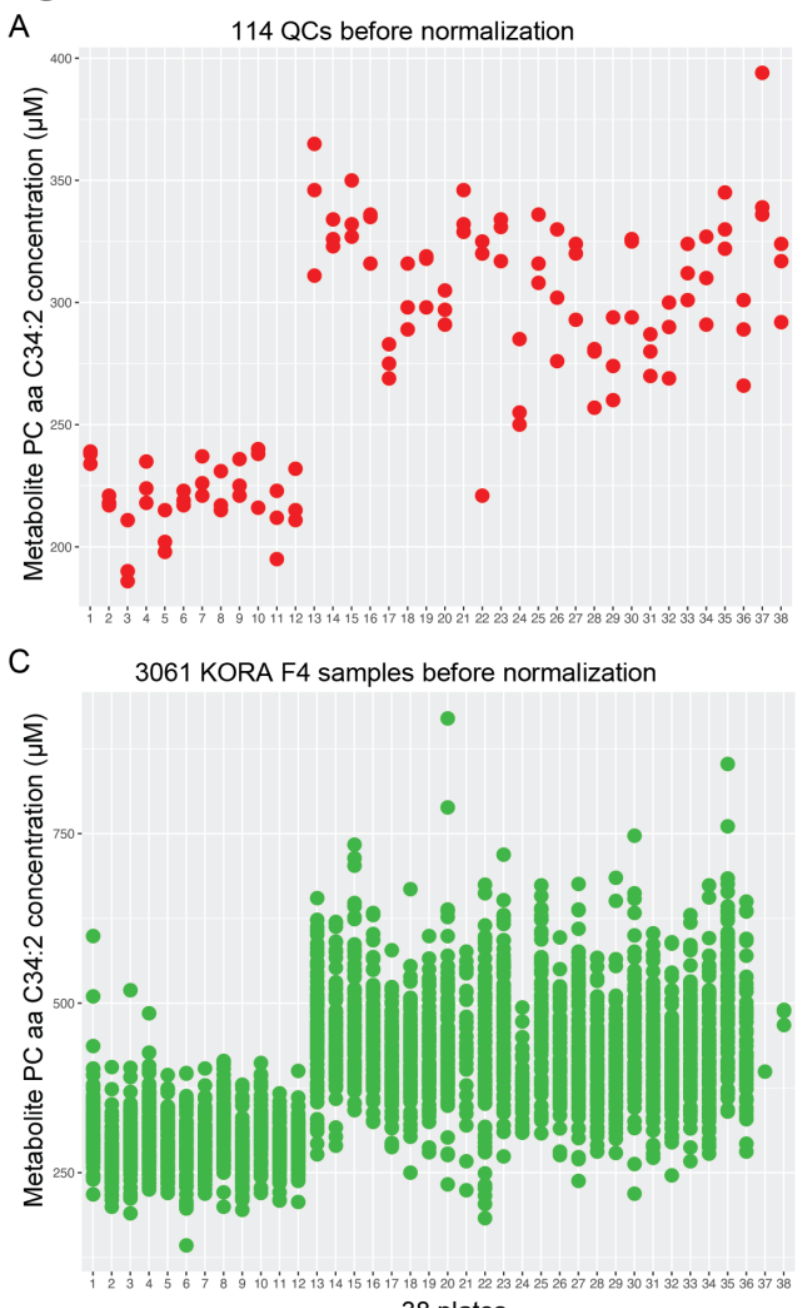

38 plates

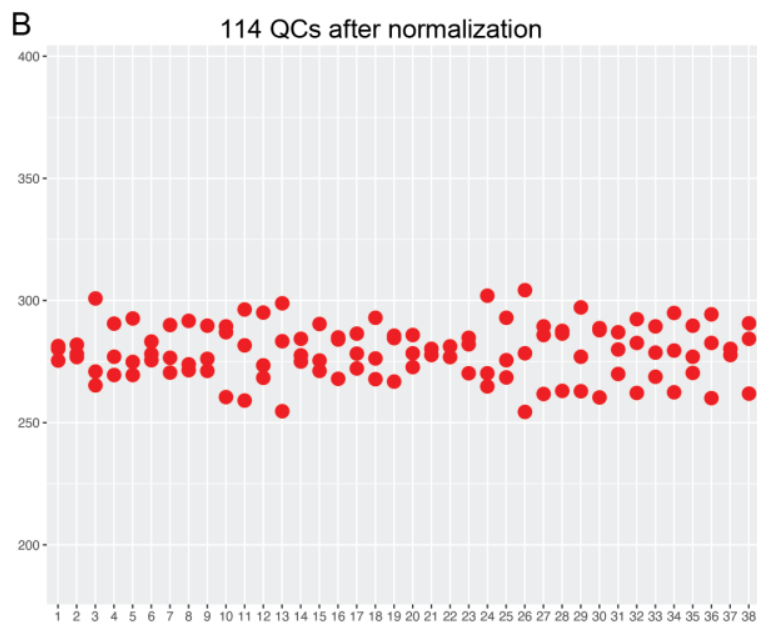

D 3061 KORA F4 samples after normalization

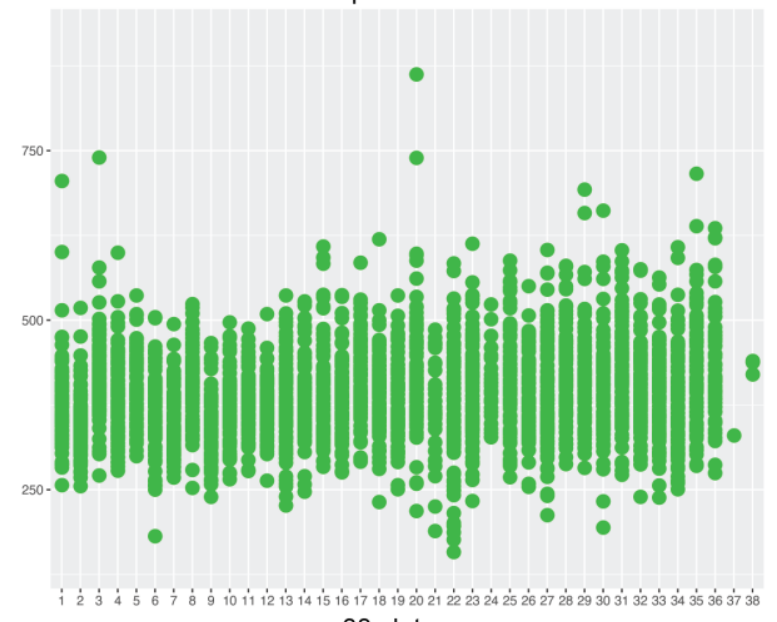

38 plates 


\section{Figure S2. Correlation of nine sphingomyelins in $\mathbf{3 8 5}$ hyperglycemic participants}

Pearson's correlation coefficients values of nine sphingomyelins (SMs) in 385 participants with prediabetes and T2D are shown. Both the size of the cycle and intensity of color indicate the degree of correlation between the metabolites. The numeric values of Pearson's correlation coefficients are shown in the bottom triangle.

Fig. S2

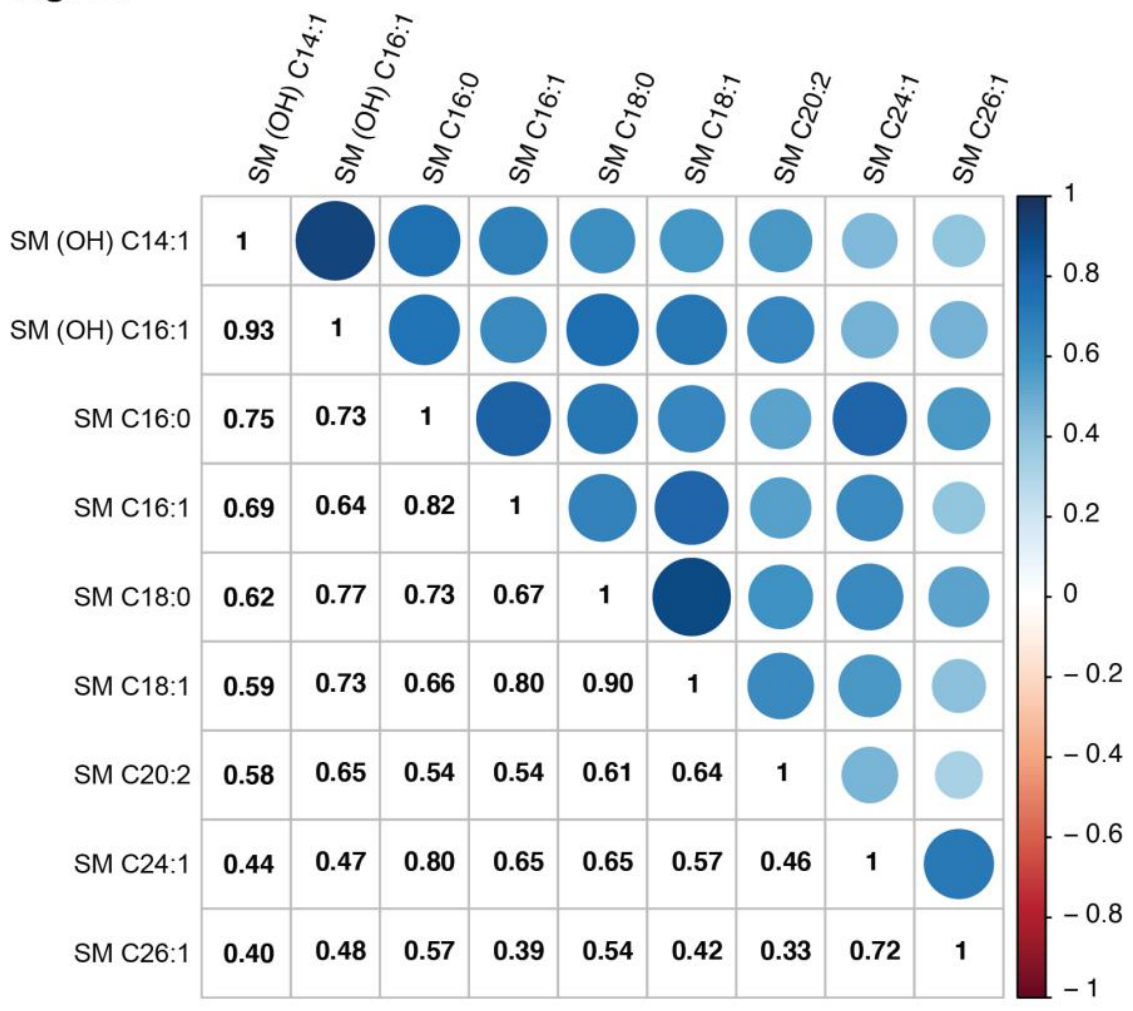

